\title{
Vortex solution in elliptic coordinates
}

\author{
Wladimir Lyra ${ }^{1}$ \\ ${ }^{1}$ New Mexico State University, Department of Astronomy, PO Box 30001 MSC 4500, Las Cruces, NM 88001, USA
}

\begin{abstract}
Vortices (flows with closed elliptic streamlines) are exact nonlinear solutions to the compressible Euler equation. In this contribution, we use differential geometry to derive the transformations between Cartesian and elliptic coordinates, and show that in elliptic coordinates a constant vorticity flow reduces to $\dot{\mu}=0$ and $\dot{v}=$ const along the streamline $\mu_{0}$ that matches the vortex eccentricity.
\end{abstract}

\section{INTRODUCTION}

Vortices are important for planet formation, theorized as favorable locations for dust trapping (Barge \& Sommeria 1995). Crescent-shaped asymmetries have been observed in sub-mm images of protoplanetary disks (van der Marel et al. 2013), although their unambiguous identification vortices has been elusive. A patch of constant vorticity follows the solution $\boldsymbol{u}=\Omega y / \chi \hat{x},-x \chi \hat{y}$, where $(x, y)$ are the Cartesian coordinates, $\Omega$ is a constant and $\chi=x / y>1$ is the vortex aspect ratio. Given the elliptic streamlines, a solution in terms of elliptic coordinates $(\mu, v)$ is desirable.

\section{ELLIPTICAL COORDINATES}

The orthogonal elliptical coordinate system is

$$
\begin{aligned}
& x=f \cosh \mu \cos v, \\
& y=f \sinh \mu \sin v,
\end{aligned}
$$

where $f=a \epsilon$ is the focal length, $a$ the semi-major axis, and $\epsilon$ the eccentricity. Constant $\mu$ define ellipses, constant $v$ define hyperbolae. The coordinates describe confocal ellipses: the focal distance is constant, so changing $\mu$ changes not only the semimajor axis but also the eccentricity.

\section{Metric}

The metric of this system is

$$
\begin{aligned}
g_{i j} & =\frac{\partial x^{\alpha}}{\partial q^{i}} \frac{\partial x^{\beta}}{\partial q^{j}} g_{\alpha \beta}, \\
& =f^{2}\left(\sinh ^{2} \mu+\sin ^{2} v\right) \delta_{i j} .
\end{aligned}
$$

where $x=(x, y)$ and $\boldsymbol{q}=(\mu, v)$ are Cartesian and elliptic coordinates; $g_{\alpha \beta}=\delta_{\alpha \beta}$ is the metric of Cartesian space. From this transformation, it follows that the scale factors are equal

$$
\begin{aligned}
& h_{\mu}=\sqrt{g_{\mu \mu}}=f \sqrt{\sinh ^{2} \mu+\sin ^{2} v}, \\
& h_{v}=\sqrt{g_{v v}}=f \sqrt{\sinh ^{2} \mu+\sin ^{2} v} .
\end{aligned}
$$

We hereafter use $h=h_{\mu}=h_{v}$. We also use the equivalent definition

$$
h=\frac{f}{\sqrt{2}} \sqrt{\cosh 2 \mu-\cos 2 v} .
$$


The derivatives with respect to the coordinates are

$$
\begin{aligned}
& \partial_{\mu} h=\frac{f^{2}}{2 h} \sinh 2 \mu, \\
& \partial_{\nu} h=\frac{f^{2}}{2 h} \sin 2 v .
\end{aligned}
$$

We calculate the Christoffel symbols in non-coordinate basis

$$
\begin{aligned}
\Gamma_{\hat{\alpha} \hat{\beta} \hat{\gamma}} & =\frac{1}{2}\left(c_{\hat{\alpha} \hat{\beta} \hat{\gamma}}+c_{\hat{\alpha} \hat{\gamma} \hat{\beta}}-c_{\hat{\beta} \hat{\gamma} \hat{\alpha}}\right), \\
\Gamma_{\hat{\beta} \hat{\gamma}}^{\hat{\alpha}} & =g^{\hat{\alpha} \hat{\zeta}} \Gamma_{\hat{\zeta} \hat{\beta} \hat{\gamma}},
\end{aligned}
$$

where $c_{\hat{\beta} \hat{\gamma} \hat{\alpha}}=g_{\hat{\alpha} \hat{\zeta}} c_{\hat{\beta} \hat{\gamma}} \hat{\zeta}$ are the connection coefficients, given by

$$
\left[e_{\hat{\beta}}, e_{\hat{\gamma}}\right]=c_{\hat{\beta} \hat{\gamma}}^{\hat{\alpha}} \partial_{\hat{\alpha}} .
$$

Given that $e_{\hat{\mu}}=h^{-1} \partial_{\mu}$ and $e_{\hat{v}}=h^{-1} \partial_{v}$, we have

$$
\begin{aligned}
{\left[e_{\hat{\mu}}, e_{\hat{v}}\right] } & =\frac{1}{h}\left[\frac{\partial}{\partial \mu}\left(\frac{1}{h} \frac{\partial}{\partial v}\right)-\frac{\partial}{\partial v}\left(\frac{1}{h} \frac{\partial}{\partial \mu}\right)\right] \\
& =\frac{f^{2}}{2 h^{4}}\left(\sin 2 v \frac{\partial}{\partial \mu}-\sinh 2 \mu \frac{\partial}{\partial v}\right) \\
& =\frac{f^{2}}{2 h^{3}}\left(\sin 2 v \partial_{\hat{\mu}}-\sinh 2 \mu \partial_{\hat{v}}\right) \\
& =-\left[e_{\hat{v}}, e_{\hat{\mu}}\right] .
\end{aligned}
$$

The connection coefficients are thus

$$
\begin{aligned}
& c_{\hat{\mu} \hat{v}}^{\hat{\mu}}=-c_{\hat{v} \hat{\mu}} \hat{\mu}=\frac{f^{2}}{2 h^{3}} \sin 2 v, \\
& c_{\hat{\mu} \hat{v}} \hat{v}=-c_{\hat{v} \hat{\mu}} \hat{v}=-\frac{f^{2}}{2 h^{3}} \sinh 2 \mu .
\end{aligned}
$$

And the Christoffel symbols are

$$
\begin{aligned}
\Gamma_{\hat{\nu} \hat{\mu}}^{\hat{\mu}} & =-\Gamma_{\hat{\mu} \hat{\mu}}^{\hat{v}}=\frac{f^{2}}{2 h^{3}} \sin 2 v, \\
\Gamma_{\hat{\mu} \hat{v}}^{\hat{v}} & =-\Gamma_{\hat{\nu} \hat{v}}^{\hat{\mu}}=\frac{f^{2}}{2 h^{3}} \sinh 2 \mu .
\end{aligned}
$$

The elliptic and Cartesian unit vectors $\hat{e}_{i}$ and $\hat{x}_{i}$ transform according to

$$
\hat{e}_{i}=\frac{1}{h_{i}} \frac{\partial x_{j}}{\partial e_{i}} \hat{x}_{j}
$$

i.e.,

$$
\begin{aligned}
{\left[\begin{array}{c}
\hat{\mu} \\
\hat{v}
\end{array}\right] } & =\left[\begin{array}{ll}
h_{\mu}^{-1} \partial_{\mu} x & h_{\mu}^{-1} \partial_{\mu} y \\
h_{v}^{-1} \partial_{\nu} x & h_{v}^{-1} \partial_{\nu} y
\end{array}\right]\left[\begin{array}{l}
\hat{x} \\
\hat{y}
\end{array}\right] \\
& =\frac{f}{h(\mu, v)}\left[\begin{array}{rr}
\sinh \mu \cos v & \cosh \mu \sin v \\
-\cosh \mu \sin v & \sinh \mu \cos v
\end{array}\right]\left[\begin{array}{l}
\hat{x} \\
\hat{y}
\end{array}\right]
\end{aligned}
$$


This can be written compactly as

$$
\hat{e}_{i}=E_{i j} \hat{x}_{j}
$$

where $E_{i j}$ is the elliptic rotation matrix. Its inverse is

$$
E^{-1}=\frac{f}{h}\left[\begin{array}{rr}
\sinh \mu \cos v & -\cosh \mu \sin v \\
\cosh \mu \sin v & \sinh \mu \cos v
\end{array}\right] .
$$

The velocity is

$$
u_{i}=h_{i} \dot{q}_{i} \hat{q}_{i}
$$

which means

$$
\begin{aligned}
\boldsymbol{u} & =\dot{x} \hat{x}+\dot{y} \hat{y}, \\
& =h_{\mu} \dot{\mu} \hat{\mu}+h_{\nu} \dot{v} \hat{v} .
\end{aligned}
$$

We can also get the velocity by the rotation matrix

$$
u_{\hat{e}_{i}}=E_{i j} u_{c_{j}}
$$

i.e.,

$$
\begin{gathered}
u_{\hat{\mu}}=E_{11} u_{x}+E_{12} u_{y}, \\
u_{\hat{v}}=E_{21} u_{x}+E_{22} u_{y} .
\end{gathered}
$$

Yielding the variation of the coordinate bases

$$
\begin{aligned}
& \dot{\mu}=f h^{-2}\left(\sinh \mu \cos v u_{x}+\cosh \mu \sin v u_{y}\right) \text {, } \\
& \dot{v}=f h^{-2}\left(-\cosh \mu \sin v u_{x}+\sinh \mu \cos v u_{y}\right) \text {. } \\
& \text { VORTEX MOTION }
\end{aligned}
$$

Consider a vortex in Cartesian coordinates

$$
\begin{aligned}
& u_{x}=-\Omega y \chi \\
& u_{y}=\Omega x / \chi
\end{aligned}
$$

We seek to transform this into elliptic coordinates. The vortex motion occurs on ellipses of constant eccentricity, whereas the elliptic coordinate system defines confocal ellipses of different eccentricity. An elliptic coordinate system based on constant eccentricity (Chang \& Oishi 2010), although matching the flow geometry, is not orthogonal, which complicates analysis (Lyra \& Lin 2013). If the streamlines matched the eccentricities of the confocal ellipses, the velocity would everywhere reduce to $\dot{\mu}=0$ and $\dot{v}=$ const. However, that is not the case, as one can verify that this is not divergenceless. In fact, there is only one streamline that obeys $\dot{\mu}=0$ and $\dot{v}=$ const, which is the streamline of eccentricity matching the eccentricity of the vortex. This is the particular ellipse $\mu_{0}$, given by $\tanh \mu_{0}=\chi^{-1}$. We write the velocities as

$$
\begin{aligned}
& u_{x}=-\Omega f \cosh \mu \sin v, \\
& u_{y}=\Omega f \sinh \mu \cos \nu .
\end{aligned}
$$


We transform these into elliptical coordinates by the rotation matrix

yielding

$$
u_{\hat{e}_{i}}=E_{i j} u_{c_{j}}
$$

$$
\begin{aligned}
& u_{\hat{\mu}}=-\Omega \frac{f^{2}}{2 h}\left(\frac{\cosh 2 \mu-\cosh 2 \mu_{0}}{\sinh 2 \mu_{0}}\right) \sin 2 v, \\
& u_{\hat{v}}=\Omega \frac{f^{2}}{2 h}\left(\frac{\sinh 2 \mu}{\sinh 2 \mu_{0}}\right)\left(\cosh 2 \mu_{0}-\cos 2 v\right) .
\end{aligned}
$$

The divergence is

$$
\begin{aligned}
\nabla \cdot A & =u_{; \hat{\alpha}}^{\hat{\alpha}}=u_{, \hat{\alpha}}^{\hat{\alpha}}+\Gamma_{\hat{\beta} \hat{\alpha}}^{\hat{\alpha}} u^{\hat{\beta}}, \\
& =u_{, \hat{\alpha}}^{\hat{\alpha}}+\frac{f^{2}}{2 h^{3}}\left(u^{\hat{\mu}} \sin 2 v+u^{\hat{v}} \sinh 2 \mu\right),
\end{aligned}
$$

or, abandoning the co-variant formulation,

$$
\nabla \cdot A=\frac{1}{h^{2}}\left(\frac{\partial h u_{\hat{\mu}}}{\partial \mu}+\frac{\partial h u_{\hat{v}}}{\partial v}\right) .
$$

we conclude that the flow is divergenceless.

Eq. (33) and Eq. (34) may seem daunting at first, but following the motion at the ellipse of $\mu=\mu_{0}$ simplifies it considerably. For $\mu=\mu_{0}$, Eq. (33) cancels. For Eq. (34), the factor in parentheses becomes unity; the next term, given Eq. (6), is $2 h / f^{2}$. Thus, for $\mu=\mu_{0}$, the motion is $u_{\hat{\mu}}=0, u_{\hat{v}}=\Omega h_{0}$. Comparing with Eq. (22) yields

$$
\begin{aligned}
& \dot{\mu}=0, \\
& \dot{v}=\Omega .
\end{aligned}
$$

For the particular $\mu=\mu_{0}$ ellipse, the motion has constant $\mu$ : a closed elliptic streamline. The angle $v$ rotates uniformly. Notice that this does not mean that the velocity itself is uniform, since $h$ depends on $v$. The explicit dependency of $u_{v}$ on $v$ is

$$
\begin{aligned}
u_{v}^{2} & =\frac{\Omega^{2} f^{2}}{2}(\cosh 2 \mu-\cos 2 v) \\
& =\Omega^{2} f^{2}\left(\sinh ^{2} \mu+\sin ^{2} v\right) .
\end{aligned}
$$

\section{ENERGY CONSERVATION}

That the kinetic energy $K=u_{v}^{2} / 2$ depends on $v$, a function of time, may seem strange at first. We show that this happens because the velocity change is compensated by a change in pressure $(p)$, conserving the total energy. The energy equation is

$$
\frac{\partial E}{\partial t}=-\nabla \cdot[\boldsymbol{u}(E+p)]+\boldsymbol{F}_{b} \cdot \boldsymbol{u}
$$

where $\boldsymbol{F}_{b}$ is a body force. The total energy is $E=K+\varepsilon$, where and $\varepsilon=k_{B} T$ is the internal energy ( $k_{B}$ is Boltzmann's constant and $T$ is the temperature). In the absence of a body force and for constant temperature, this reduces to

$$
\frac{\partial}{\partial t}\left(\frac{u^{2}}{2}\right)=-(\boldsymbol{u} \cdot \nabla)\left(\frac{u^{2}}{2}+p / \rho\right)
$$


therefore

$$
\frac{d}{d t}\left(\frac{u^{2}}{2}\right)=-(\boldsymbol{u} \cdot \nabla) p / \rho
$$

The enthalpy is found by Euler's equation

$$
\begin{aligned}
& \partial_{x} p / \rho=-u_{x} \partial_{x} u_{y}=\Omega^{2} x \\
& \partial_{y} p / \rho=-u_{y} \partial_{y} u_{x}=\Omega^{2} y
\end{aligned}
$$

Taking the $x$-derivative above and the $y$ below, we find $\nabla^{2} p / \rho=\Omega^{2}$; therefore

$$
p / \rho=\frac{1}{2} \Omega^{2}\left(x^{2}+y^{2}\right)+C
$$

which is an intriguing result: an incompressible elliptical vortex produces an axis-symmetric pressure distribution. Transforming into elliptic coordinates and eliminating the constant

$$
p / \rho=\frac{1}{2} \Omega^{2} f^{2}\left(\cosh ^{2} \mu \cos ^{2} v+\sin ^{2} \mu \sin ^{2} v\right) .
$$

Along the $\mu_{0}$ streamline, the advection reduces to the $v$-term

$$
\begin{aligned}
-(\boldsymbol{u} \cdot \nabla) P / \rho & =-u_{v} h^{-1} \partial_{\nu} p / \rho \\
& =\frac{1}{2} \Omega^{3} f^{2} \sin 2 v,
\end{aligned}
$$

whereas the time derivative of the kinetic energy is

$$
\begin{aligned}
\frac{d}{d t}\left(\frac{u^{2}}{2}\right) & =\Omega^{2} h \frac{d h}{d t} \\
& =\frac{1}{2} \Omega^{3} f^{2} \sin 2 v .
\end{aligned}
$$

That the two variations match amounts to conservation of energy: along the ellipse, the material slows down or speeds up in order to match the pressure variation.

\section{EULER EQUATION IN ELLIPTICAL COORDINATES}

We consider now the force balance. We use the transformations here derived to write the Euler equation in elliptic coordinates

$$
\partial_{t} \boldsymbol{u}+(\boldsymbol{u} \cdot \nabla) \boldsymbol{u}=-\nabla p / \rho
$$

Using covariant derivatives, this reads

$$
\partial_{t} u^{\hat{k}}+u^{\hat{p}} \partial_{\hat{p}} u^{\hat{k}}+\Gamma_{\hat{m} \hat{n}}^{\hat{k}} u^{\hat{m}} u^{\hat{n}}=-\partial_{\hat{k}} p / \rho .
$$

For $\mu$, the correction due to the Christoffel symbols is

$$
\begin{aligned}
\Gamma_{\hat{m} \hat{n}}^{\hat{\mu}} u^{\hat{m}} u^{\hat{n}} & =\Gamma_{\hat{v} \hat{u}^{\prime}}^{\hat{\mu}} \\
& =\frac{f^{2}}{2 h^{3}}\left[u^{\hat{v}} u^{\hat{\mu}} \sin 2 v-\left(u^{\hat{v}}\right)^{2} \sinh 2 \mu\right] .
\end{aligned}
$$

The same procedure for $v$ yields 


$$
\begin{aligned}
\Gamma_{\hat{m} \hat{n}}^{\hat{v}} u^{\hat{m}} u^{\hat{v}} & =\Gamma_{\hat{v} \hat{\mu}}^{\hat{u}} u^{\hat{\mu}} u^{\hat{v}}+\Gamma_{\hat{\mu} \hat{\mu}}^{\hat{\mu}}\left(u^{\hat{\mu}}\right)^{2}, \\
& =\frac{f^{2}}{2 h^{3}}\left[u^{\hat{v}} u^{\hat{\mu}} \sinh 2 \mu-\left(u^{\hat{\mu}}\right)^{2} \sin 2 v\right] .
\end{aligned}
$$

Abandoning the co-variant notation

$$
\begin{aligned}
& \partial_{t} u_{\mu}=-\left(h^{-1} u_{\mu} \partial_{\mu}+h^{-1} u_{v} \partial_{v}\right) u_{\mu}-h^{-1} \partial_{\mu} p / \rho-\frac{f^{2}}{2 h^{3}}\left(u_{v} u_{\mu} \sin 2 v-u_{v}^{2} \sinh 2 \mu\right), \\
& \partial_{t} u_{v}=-\left(h^{-1} u_{\mu} \partial_{\mu}+h^{-1} u_{v} \partial_{\nu}\right) u_{v}-h^{-1} \partial_{\nu} p / \rho-\frac{f^{2}}{2 h^{3}}\left(u_{v} u_{\mu} \sinh 2 \mu-u_{\mu}^{2} \sin 2 v\right) .
\end{aligned}
$$

This differs from the usual equations by the presence of the extra force

$$
\boldsymbol{F}=\frac{f^{2}}{2 h^{3}}\left[\left(u_{v} u_{\mu} \sin 2 v-u_{v}^{2} \sinh 2 \mu\right) \hat{\mu}+\left(u_{v} u_{\mu} \sinh 2 \mu-u_{\mu}^{2} \sin 2 v\right) \hat{v}\right]
$$

Contracting this force with the velocity yields $\boldsymbol{F} \cdot \boldsymbol{u}=0$, which shows that this force is inertial. For the vortical flow, again following the $\mu_{0}$ streamline where $\dot{\mu}=0$ and $\dot{v}=\Omega$, these reduce to

$$
\begin{aligned}
-\frac{\Omega^{2} f^{2}}{2} \sinh 2 \mu_{0} & =-\partial_{\mu} p / \rho \\
\left(u_{v} \partial_{v}\right) u_{v} & =-\partial_{\nu} p / \rho
\end{aligned}
$$

I.e, a constant centrifugal force that balances the normal pressure gradient, and inertia in the tangential direction exchanging kinetic energy with the pressure field. Fig 1 sketches the forces.

\section{REFERENCES}

Barge, P., \& Sommeria, J. 1995, A\&A, 295, L1

Chang, P., \& Oishi, J. S. 2010, ApJ, 721, 1593, doi: 10.1088/0004-637X/721/2/1593

Lyra, W., \& Lin, M.-K. 2013, ApJ, 775, 17, doi: $10.1088 / 0004-637 X / 775 / 1 / 17$ van der Marel, N., van Dishoeck, E. F., Bruderer, S., et al. 2013, Science, 340, 1199, doi: 10.1126/science.1236770 


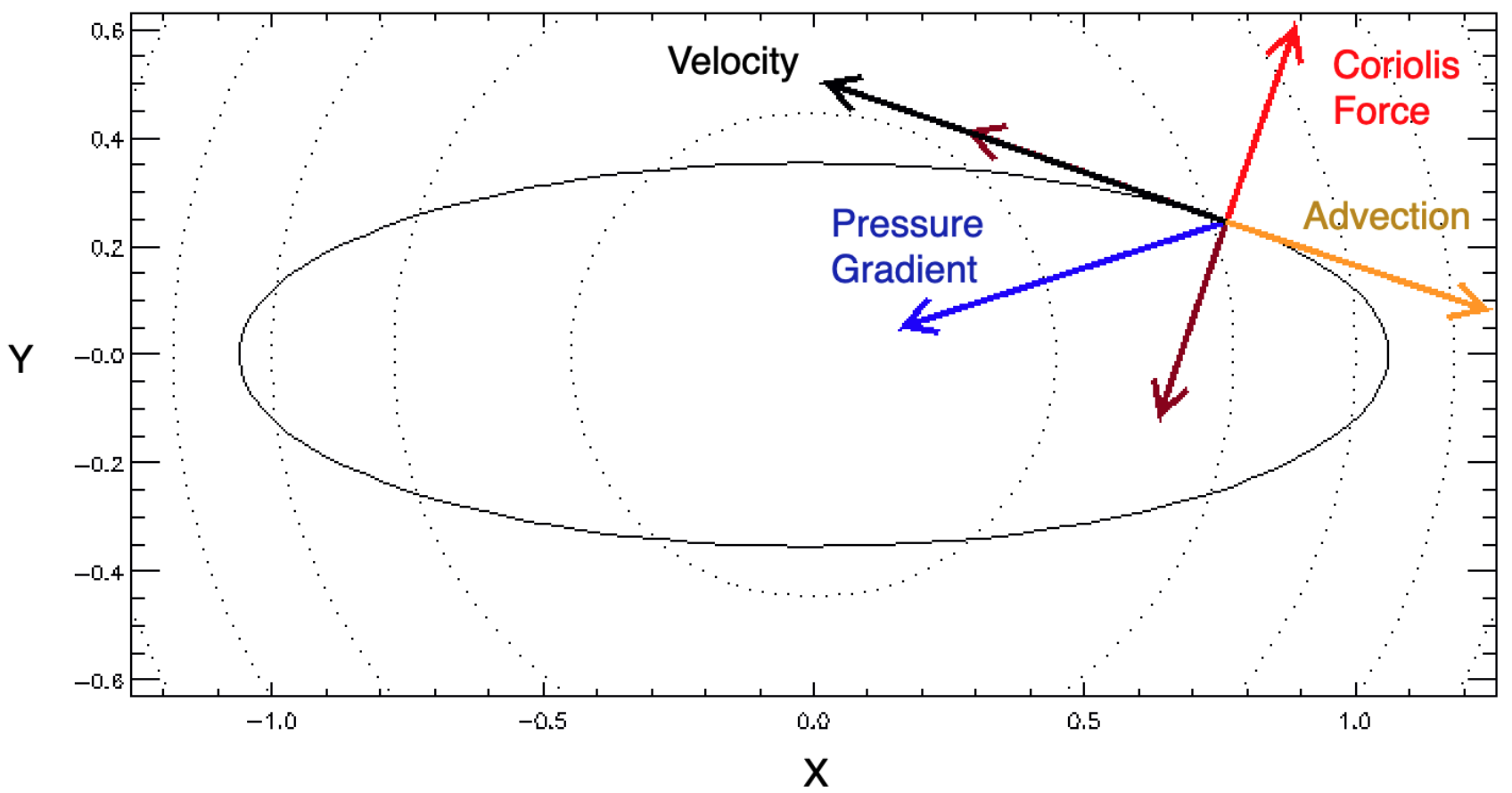

Figure 1. Force balance in an elliptic vortex streamline (solid line). Dotted circles represent the pressure contours. The velocity (black arrow) is tangent to the streamline, in the $\hat{v}$ direction. The pressure gradient (blue arrow) is broken down in its $\hat{\mu}$ and $\hat{v}$ components (brown arrows). The $\hat{\mu}$ component is balanced by the centrifugal force (red arrow); the $\hat{v}$ component is balanced by advection. 